\title{
AiMT
}

Advances in Military Technology

Vol. 15, No. 2, 2020, pp. 317-328

ISSN 1802-2308, eISSN 2533-4123

DOI 10.3849/aimt.01375

\section{The Causes of Leakage Deviations from sinc Function in DFT and Ways to Minimize Them}

\author{
K. Hajek ${ }^{*}$ \\ Department of Electrical Engineering, University of Defence in Brno, Czech Republic
}

The manuscript was received on 17 January 2020 and was accepted after revision for publication as research paper on 7 September 2020.

\begin{abstract}
:
The article describes a new model for the solution of the instability problem of discrete Fourier transform (DFT) spectra leakage for the incoherent real sinusoidal signal for solving the most accurate estimation of its frequency. This paper shows this phenomenon by a new form of DFT spectrum expression. It consists mainly of the spectrum of the rectangular signal in the form of the sinc function, modulated to the frequency of the tested signal in baseband. To do this, analogous spectra from other neighboring bands formed by the sampling effect are added as aliasing. The analysis shows some ways to minimize or correct it. The paper shows a simpler and substantially lesser effect of aliasing in the DFT spectrum for a complex valued sinusoidal signal.
\end{abstract}

\section{Keywords:}

aliasing, DFT, frequency estimation, leakage

\section{Introduction}

DFT or its fast variant FFT is a widely used instrument for spectral analysis in many branches. In military applications, this instrument is used by radar technology, by communication technology, for evaluation of the Doppler frequency changes of GPS receivers or ultrasonic signals, by sonars, etc.

Its use is very accurate when the analyzed discrete signal is periodic and coherent with DFT. However, for a common incoherent signal this condition cannot usually be fulfilled. In this case, the result of DFT spectral analysis is not accurate. The main effect of this error is known as leakage. It is a widely studied and published problem, for example in [1-4]. The exact value of this error is usually not evaluated, the effect is only minimized using a selected window function. Yet there are tasks where we try to find the most accurate parameters of the original signal from the result of DFT, when

\footnotetext{
* Corresponding author: Department of Electrical Engineering, University of Defence in Brno, Kounicova 156/65, CZ-662 10 Brno, Czech Republic. Phone: +420 776298 417, E-mail: karel.hajek@unob.cz.ORCID 0000-0002-6362-7439
} 
we are interested in a correct evaluation of the DFT analysis error. The attempt to estimate as accurately as possible the frequency of the harmonic component of the signal under testing is a typical example of this problem. This is dealt with in many publications, such as [5-21].

\section{Model for DFT Error Analysis}

To analyze these problems, we use the real valued sinusoidal signal $s(t)$ as a typical signal to demonstrate and describe the leakage effect. Let us consider the unit amplitude $A$, the frequency $F_{0}$ and the initial phase $\varphi_{0}$. The signal frequency $F_{0}$ corresponds to the period $T_{0}=1 / F_{0}$. The sampled signal can be expressed by Eq. (1):

$$
s(n)=A \cos \left(2 \pi F_{0} n T_{\mathrm{S}}+\varphi_{0}\right)
$$

where $n$ are integer and $T_{\mathrm{S}}$ is the sampling period corresponding to the sampling frequency $F_{\mathrm{S}}=1 / T_{\mathrm{S}}$. For the DFT calculation, we consider the use of $N$ points of the discrete signal thus created, as shown in Fig. 1 for $N=16$. The choice of the $N$ value defines the formal period of the $1^{\text {st }}$ harmonic component of DFT as $T=N T_{\mathrm{S}}$ and thus the frequency of this $1^{\text {st }}$ harmonic component is given by the relation $F=1 / T$. Simultaneously, this value is the basic step of the discrete frequency axis $m F$ of the DFT calculated spectrum. Often, it is preferable to use only the relative discrete frequency values $m$, which are usually referred to as the bin unit. The relative value of the sampling frequency is then $N$.

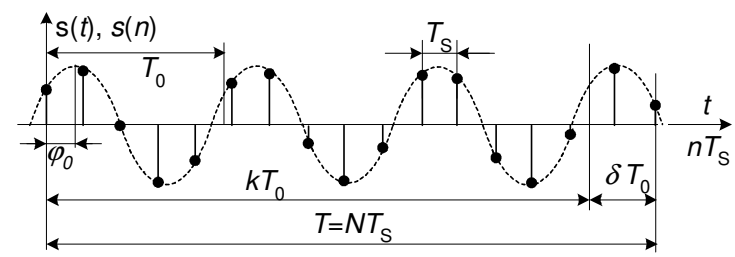

Fig. 1 Time dependency of sinusoidal signal and the accordant discretized incoherent signal in the DFT window

The aforementioned DFT spectrum error does not exist if the discrete signal periodicity condition is satisfied and if the condition $T=k T_{0}$ or $N T_{\mathrm{S}}=k T_{0}$ is met, where $k$ is an integer which expresses the number of periods of the $s(n)$ signal in the period $T$ ( $\delta=0$ in Fig. 1). For the frequency axis it holds $k F=F_{0}$ or $k F_{\mathrm{S}}=N F_{0}$. In this case, the input signal is coherent with DFT, and the resulting spectrum consists of only one spectral component with the frequency $F_{0}$ or $k_{0}$, see Fig. 2 a.
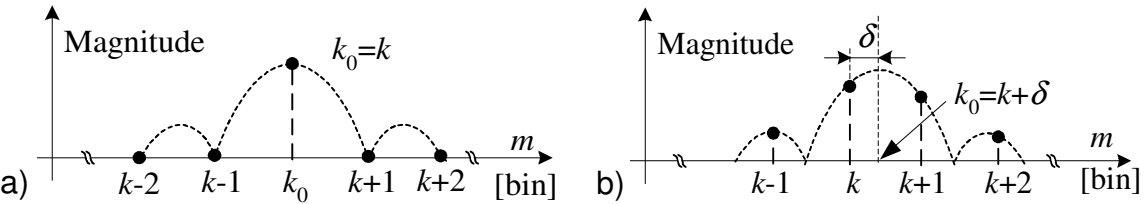

Fig. 2 DFT spectrum of amplitudes in the relative frequency axis:

a) for coherent, $b$ ) for incoherent sinusoidal signal

We obtain incoherent signal if the condition of coherence is not fulfilled. The true frequency of the $F_{0}$ signal is located between the two discrete values of the DFT 
frequency axis. This corresponds to a real number of the relative value of the frequency $k_{0}$ of the input signal. The relative frequency of the input signal can be expressed as $k_{0}=k+\delta$, where the frequency displacement $\delta$ is in the interval $-0.5<\delta<0.5$, see Fig. $2 b$.

DFT spectral analysis brings the already mentioned leakage effect in this test of incoherent sinusoidal signal ( $k_{0}$ is real number, $\delta \neq 0$ ). It calculates the erroneous, infinitely wide spectrum of harmonic components symmetrically spaced around the frequency $F_{0}$ or $k_{0}$. Their magnitudes correspond to the envelope function $\sin (x) / x=$ $\operatorname{sinc}(x)$, as shown in Fig. $2 b$ and published in many references. Note that the DFT values in this article are multiplied by $2 / N$ so that the size of the spectrum components corresponds to the amplitudes of the time sinusoidal signals (as in the Fourier series), which is physically more appropriate and more often used in practice.

\section{Deviations of DFT Spectrum from sinc Function}

The computed magnitude of the DFT spectrum of the real incoherent sinusoidal signal diverges slightly from the ideal sinc function in various ways. In addition to the $F_{0} / F_{\mathrm{S}}$ ratio, these deviations are caused by the change in the phase shift $\varphi_{0}$ of the tested signal DFT window origin [14]. Depending on the value of $\varphi_{0}$, the magnitudes of the spectrum can be changed so that the value of some of the components can drop to zero (decrease by up to $100 \mathrm{dBV}$ ), see Fig. 3.

The shape of the envelope function also depends on the value $\delta$. For example, if $\delta=0.5$, the two adjacent highest components should have the same amplitude and the amplitude spectrum should be exactly symmetric around $k_{0}$. However, in many cases this is not true, see Figs $3 \mathrm{a}$ and $3 \mathrm{~b}$, where the phase changes $\left(20^{\circ}\right.$ and $\left.110^{\circ}\right)$ have turned the asymmetry direction even for the both highest value components. On the other hand, for phase $\varphi_{0}=65^{\circ}$ as the center between the two cases, the shape of the spectrum maxima is almost symmetrical and closest to the sinc function (Fig. 3c). Therefore, the dependence of the deviation error $\delta$ on the phase clearly confirms these conclusions, see Fig. 3d. This example illustrates the effect of the phase of the tested signal on the modulus of DFT spectrum.
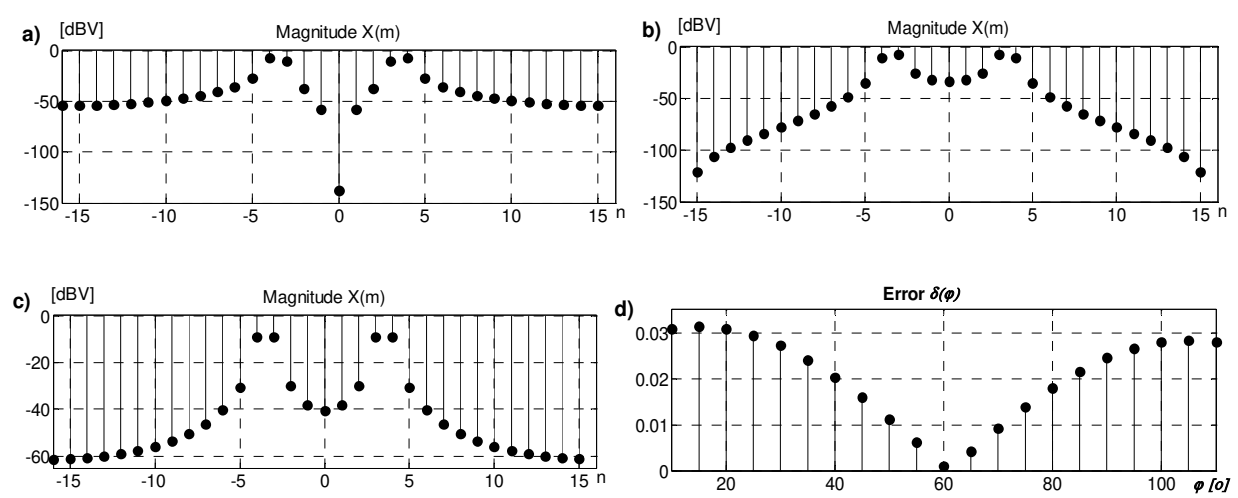

Fig. 3 Effect of phase change on DFT spectrum of sampled sinusoidal signal $\left.\left.\left(F_{0}=3.5 \mathrm{~Hz}, T=1 \mathrm{~s}, N=32, F_{\mathrm{S}}=32 \mathrm{~Hz}\right): a\right) \varphi_{0}=20^{\circ}, b\right) \varphi_{0}=110^{\circ}$

c) $\left.\varphi_{0}=65^{\circ}, d\right)$ error of $\delta v s . \varphi_{0}$ 
Aliasing is the basic cause of the deviation variability from the magnitude of the sinc function. In addition to the aliasing associated with the sampling frequency, there is yet another aliasing, analogous to the aliasing of binary data modulations. The problem is illustrated in Fig. 4. Here, the procedure for creating $N$ samples for DFT is transposed for the sake of clarity by first modulating the rectangular signal of the DFT window to a carrier continuous real valued sinusoidal signal with frequency $F_{0}$. It is obvious that the continuous and infinite complex valued spectrum function of the rectangular pulse (Fig. 4f) is added to the positive and negative frequency components $\left(-F_{0}, F_{0}\right)$ in this modulation, which corresponds to the convolution of both input spectra. This effect is known as image or negative spectral components of real signal. For this reason, they are mutually aliased as shown in Fig. 4h).

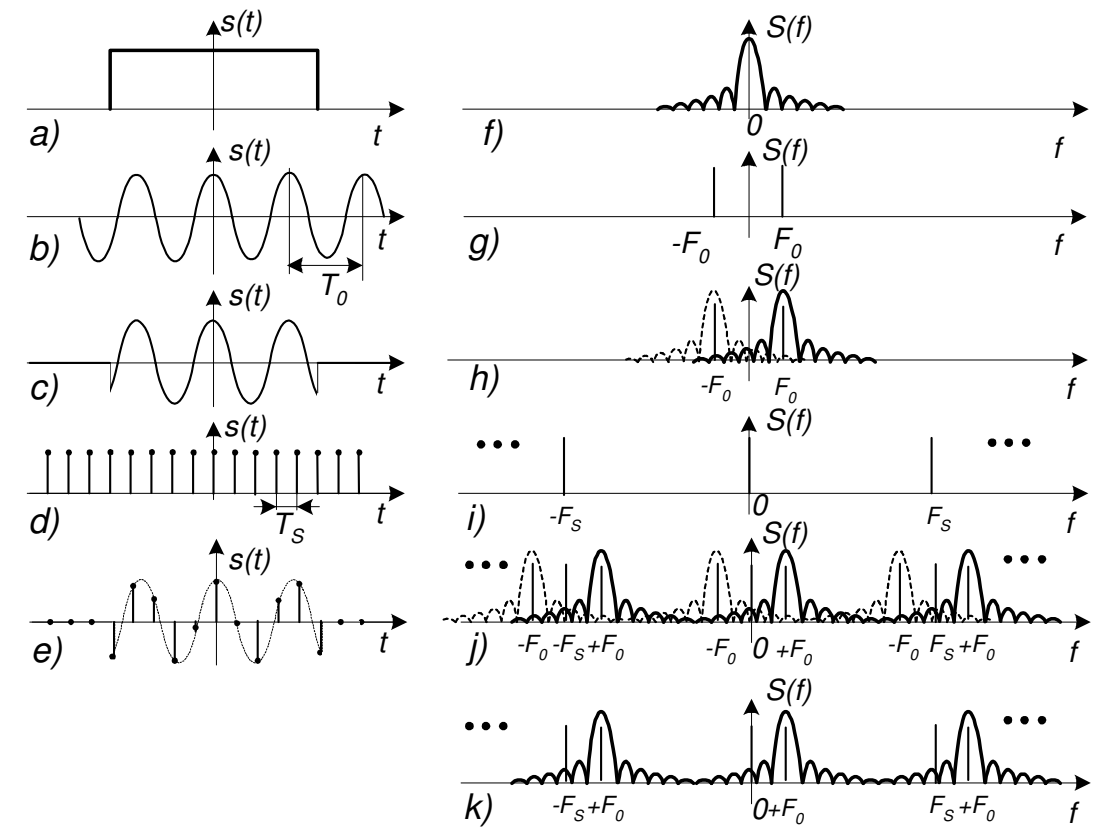

Fig. $4(a)-(e)$ the time courses of the formation of $N$ signal samples for DFT, $(f)-(i)$ the corresponding spectral functions, $(j)$ the discrete spectrum envelope of the real valued sinusoidal signal, $(k)$ the discrete spectrum envelope of the complex valued sinusoidal signal

The continuous signal is sampled by the frequency $F_{\mathrm{S}}$ (Fig. $4 \mathrm{~d}$ ) in the last step (Fig. 4e). The resulting discrete time signal is then equivalent to that of $N$ samples for the DFT spectrum obtained by the standard rectangular windowing. The accordant continuous spectral function thus goes through the convolution with the spectrum of the sampling signal (Fig. 4i) in the discrete spectrum, which is repeated for each multiple of $y F_{\mathrm{S}}$, where $y$ is integer. Since the spectral function (Fig. $4 \mathrm{~h}$ ) exceeds the frequency $F_{\mathrm{S}} / 2$, the sampling theorem is not fulfilled here and classical aliasing takes place, see Fig. $4 \mathrm{j}$, where the envelopes of discrete spectra are drawn.

Thus it is obvious that DFT adds to the spectrum of a rectangular signal, modulated to the frequency $F_{0}$ in the baseband 0 to $F_{\mathrm{S}} / 2$, also aliasing from the bands for the 
multiples of the sampling frequency, both for the $-y F_{\mathrm{S}}$ of the negative frequency halfaxis and also for the bands with positive $y F_{\mathrm{S}}$ frequencies. However, it is apparent that the greatest effect of aliasing will come from the two adjacent bands.

Another notable feature is the aliasing dependence on the relative position of the partial spectra modulated to the frequencies $+F_{0}$ and $-F_{0}$. This aliasing dependence will therefore depend on the $F_{0} / F_{\mathrm{S}}$ value. Furthermore, it will be evident that both types of aliasing will be limited to zero for high values of $N$, because increasing $N$ and the corresponding period $T$ decreases the width of the frequency step $m$, thereby narrowing the sinc band. It is also advantageous to choose the frequency value $F_{0}$ as close as possible to $F_{\mathrm{S}} / 4$, where the effect of the sum of aliasing on both sides is minimal because it is in the middle position between them.

By contrast, the DFT spectrum of a complex valued sinusoidal signal (see Fig. 4k), which is obtained analogously, but without image sub-spectra for the $-F_{0}$ frequencies, is evidently simpler. Aliasing is manifested here only from adjacent subbands for the frequencies $+F_{0}$. Thus, this aliasing does not depend on the value of $F_{0} / F_{\mathrm{S}}$ and $\varphi_{0}$. The shape of the sub-spectra will therefore only depend on the value of $N$.

\section{Spectrum of the Rectangular Signal, Modulated at $\boldsymbol{F}_{\mathbf{0}}$}

A suitable approach to obtaining this spectrum is shown for example as Eq. (3-73) in [2], where samples of the real sinusoidal signal are substituted in the general relationship for DFT. The used editing led to two independent expressions for the "carrier" frequencies $+F_{0}$ and $-F_{0}$ or $+k_{0}$ and $-k_{0}$ (and for $\varphi_{0}=0$ ) according to Eq. (2):

$$
\begin{aligned}
X(m)=\frac{1}{2 N} \frac{\sin \left[\pi\left(m-k_{0}\right)\right]}{\sin \frac{\pi\left(m-k_{0}\right)}{N}} \exp \left\{\mathrm{j}\left[\pi\left(m-k_{0}\right)\left(1+\frac{1}{N}\right)\right]\right\}+ \\
+\frac{1}{2 N} \frac{\sin \left[\pi\left(m+k_{0}\right)\right]}{\sin \frac{\pi\left(m+k_{0}\right)}{N}} \exp \left\{\mathrm{j}\left[\pi\left(m+k_{0}\right)\left(1+\frac{1}{N}\right)\right]\right\}
\end{aligned}
$$

It should be noted that this part was omitted in the third edition of [2]. The second part of DFT spectrum (the second row of Eq. (2)) corresponds with negative spectral components of real valued signal (e.g. [6-9, 16, 19]). Under the above condition $N \rightarrow \infty$, the spectra of the rectangular window in the individual sub-bands will not influence each other by aliasing. Eq. (2) is then simplified, and the fractions will change to the sinc function. Similarly, the expression for the phase in the exponent is reduced. The spectrum in the basic band for $+F_{0}$ (e.g. in Fig. $4 \mathrm{j}$ ), including the influence of the phase shift of the signal $\varphi_{0}$, can be expressed as sinc function by Eq. (3):

$$
\begin{aligned}
& X(m)=\frac{\sin \left[\pi\left(m-k_{0}\right)\right]}{\pi\left(m-k_{0}\right)} \exp \left\{\mathrm{j}\left[\pi\left(m-k_{0}\right)-\varphi_{0}\right]\right\} \\
& X(m)=\operatorname{sinc}\left[\pi\left(m-k_{0}\right)\right] \exp \left\{\mathrm{j}\left[\pi\left(m-k_{0}\right)-\varphi_{0}\right]\right\}
\end{aligned}
$$

The modulus of Eq. (3) is a modulus of the sinc function. The alternating sign values of the sinc function phase are corrected by the expression in the exponent. The resulting phase dependence is then constant and only for frequencies higher than $F_{0}$ resp. $k_{0}$ it is shifted by $180^{\circ}$. For the value $\delta=0.5$, it is $+90^{\circ}$ and $-90^{\circ}$, and the change 
in the value $\delta$ causes an adequate phase shift in addition to module asymmetry, as shown in Fig. 5.

The shape of the envelope function also depends on the value $\delta$. For example, if $\delta=0.5$, the two adjacent highest components should have the same amplitude and the amplitude spectrum should be exactly symmetric around $k_{0}$. However, in many cases this is not true, see Fig. 3, where the phase change has turned the asymmetry direction even for the highest value components.
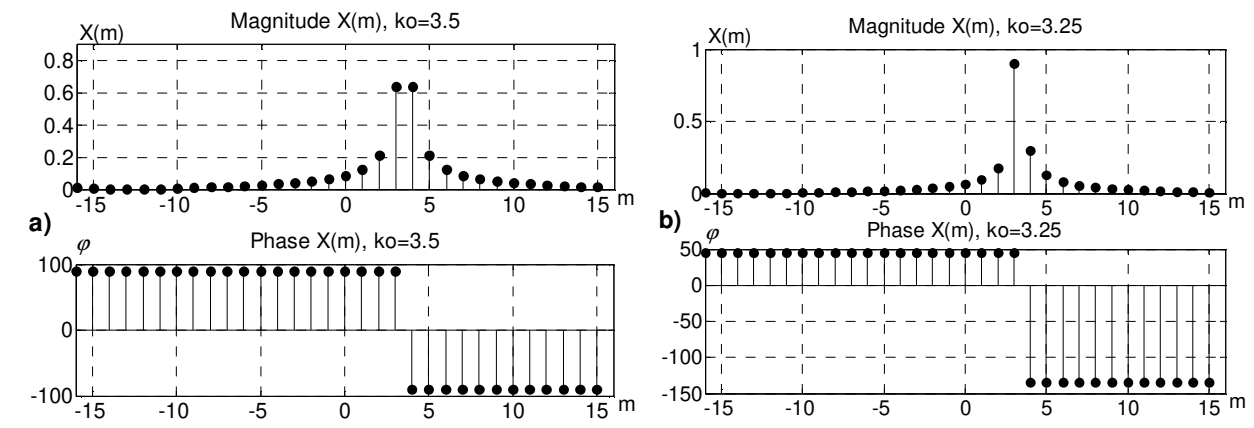

Fig. 5 Magnitude and phase of basic spectrum for sampled sinusoidal signal $\left.\left.\left(T=1 \mathrm{~s}, N=32, F_{\mathrm{S}}=32 \mathrm{~Hz}, \varphi_{0}=0^{\circ}\right): a\right) F_{0}=3.5 \mathrm{~Hz}, b\right) F_{0}=3.25 \mathrm{~Hz}$

\section{Expression of DFT as a Sum of Individual sinc Partial Spectra}

The general equation for the DFT spectrum of a real sinusoidal signal can be expressed for the finite value $N$ as the sum of the base band pairs corresponding to the frequency $k_{0}$ and $-k_{0}$ with other spectra pairs for all multiples of the relative sampling frequency $y N$, where $y$ are integers in the interval $-\infty<y<\infty$ by Eq (4):

$$
\begin{aligned}
& X(m)=\sum_{y=-\infty}^{\infty}( \operatorname{sinc}\left[\pi\left(m-y k_{0}\right)\right] \exp \left\{\mathrm{j}\left[\pi\left(m-y k_{0}\right)-\varphi_{0}\right]\right\}+ \\
&\left.\left.+\operatorname{sinc}\left[\pi\left(m+y k_{0}\right)\right] \exp \left\{\mathrm{j}\left[\pi\left(m+y k_{0}\right)+\varphi_{0}\right]\right]\right\}\right)
\end{aligned}
$$

In this way, aliasing from all other bands is reflected in the base frequency band and we obtain the DFT spectrum in a different way than according to Eq. (2). Of course, this calculation is more complicated than the calculation by Eq. (2) or by DFT, although for a deviation from DFT of less than about $10^{-3}(-60 \mathrm{~dB})$ it is sufficient to solve Eq. (4) for $y$ only in the interval $-5<y<5$.

An important conclusion is that this way of expressing DFT shows the abovementioned effect of aliasing and the influence of the phase $\varphi_{0}$ of the signal, which is the principal reason why the undefined change of the signal phase causes seemingly random changes to the DFT magnitude leakage, see Fig. 3. However, many articles on frequency estimation consider this effect as a bias of method with the cause of estimation nonlinearity, e.g. [10-12].

On the other hand, in the case of a complex sine wave, Eqs (2) and (4) can be used to express the DFT with only the first line valid for both equations: 


$$
\begin{aligned}
& X(m)=\frac{1}{2 N} \frac{\sin \left[\pi\left(m-k_{0}\right)\right]}{\sin \frac{\pi\left(m-k_{0}\right)}{N}} \exp \left\{\mathrm{j}\left[\pi\left(m-k_{0}\right)\left(1+\frac{1}{N}\right)\right]\right\} \\
& X(m)=\sum_{y=-\infty}^{\infty} \operatorname{sinc}\left[\pi\left(m-y k_{0}\right)\right] \exp \left\{\mathrm{j}\left[\pi\left(m-y k_{0}\right)-\varphi_{0}\right]\right\}
\end{aligned}
$$

This corresponds to Fig. 4k and causes only classical aliasing without the aliasing effect of the image components. Therefore, the effect of aliasing depends only on $N$ and it does not depend on $F_{0} / F_{\mathrm{S}}$ and $\varphi_{0}$. It follows that the relation for deviation $\delta$ can be expressed exactly from the three largest components of the spectrum $X_{k-1}$ to $X_{k+1}$ only as a function of $N$, with absolute accuracy. It was derived universally and with consideration of zero-padding in [15]. In the case without padding, the relation for $\delta$ can be expressed as

$$
\delta=\frac{N}{2 \pi} \arcsin \left[2 \sin \frac{\pi}{N} \operatorname{Re}(U)\right]
$$

where $U$ is expressed from the three largest components of the spectra

$$
U=\frac{X\left[k_{m}-1\right] \exp \left(\frac{\mathrm{j} \pi}{N}\right)-X\left[k_{m}+1\right] \exp \left(-\frac{\mathrm{j} \pi}{N}\right)}{2 X\left[k_{m}\right]-X\left[k_{m}+1\right]-X\left[k_{m}-1\right]}
$$

\section{Ways for Minimizing Leakage Deviations from sinc Function}

The previous analysis for a real valued sinusoidal signal has shown that the parameters $N, F_{0} / F_{\mathrm{S}}$ and $\varphi_{0}$ have a decisive influence on the DFT spectrum deviations from the sinc function and on their instability. The evaluation of these deviations is also limited by the signal to noise ratio (SNR).

The analysis clarifies that increasing the value of $N$ is the easiest way to reduce the discussed deviations. This is well known but it has practical limits. Another way results from the mentioned symmetrical dependence of deviations on the value of $F_{0} / F_{\mathrm{S}}$ in the interval $0<F_{0} / F_{\mathrm{S}}<0.5$, where the maximum deviation is at the edges of the interval and the minimum is for the value $F_{0} / F_{\mathrm{S}}=0.25$. Examples of these dependencies are given e.g. in Figs $6 a$ and $6 \mathrm{~b}$. In practice, it may be difficult to set 0.25 . Then we at least avoid the boundary values of this interval with worse results.

The use of window functions is an essential tool for minimizing the discussed deviations. It is discussed in many publications, e.g. $[15,16,18,19,21]$, but the results of these works are not unambiguous and they are even contradictory in some cases. The conclusions of the presented analysis explain why it is and what the principles for optimal use of suitable window functions should be. Obviously, the effect of the window function used depends on several parameters.

Above all, the influence of the $F_{0} / F_{\mathrm{S}}$ ratio is obvious. The value of relative distance of the maximum of the nearest neighboring sub-band is the main reason. For $F_{0} / F_{\mathrm{S}}<0.25$, it is the first negative sub-band. If this relative range is very small, aliasing from the nearest sub-band has a dominant influence on the deviations from the sinc function. Therefore, the efficiency of such a window, whose frequency dependence has a minimum transmission of aliasing components from this neighboring band, is 
increased just for the evaluated baseband components (e.g. Hann's window). In the case of larger distances (e.g. $F_{0} / F_{\mathrm{S}}>0.1$ ), the influence of distant sub-bands increases. In this case, it is desirable that the frequency dependence of attenuation of the used window be increasing (e.g. Hann's window). The effect of the near image maximum aliasing effect can be so pronounced that for small $F_{0} / F_{\mathrm{S}}$ values, the error values of deviation $\delta$, e.g. a rectangular window, are lower compared to e.g. Hann's window as shown in Figs 6c and 6d.

Similar considerations apply when we observe the degree of the deviations effect for different SNR. Obviously, for a low SNR, the effect of more distant sub-bands is below the noise level. Therefore, window functions can be used whose attenuation is greatest for the nearest sub-bands with the dominant effect above the noise level [13]. With a high SNR value, the aliasing from far sub-bands must also be considered. The width of the first sidelobe also has a similar effect [16].

These aspects can combine with each other. This explains why it is difficult to find the optimal selection of the window function so as to attenuate the transmission of aliasing from neighboring sub-bands with the most significant influence. This fact also explains the differences in the results of various publications on the selection of appropriate window functions, where the authors do not consider all the aspects and parameters resulting from the presented analysis.

A special case is the procedure where the authors used the estimated $\delta$, calculated for a rectangular window, for other types of windows. The difference from the rectangular window was compensated by a fixed correction value (e.g. [15, 20]). These procedures cannot work universally because under different conditions $\left(N, F_{0} / F_{\mathrm{S}}\right.$, SNR) the effect of window functions is different, as is evident from the presented analysis. The authors derived the respective correction coefficients for a particular case; however, for other conditions, these procedures can produce worse results.

It is appropriate to compare these relations with the frequently used Jacobsen's relation for a rectangular window $[11,20]$

$$
\delta=\operatorname{Re}\left(\frac{X_{k-1}-X_{k+1}}{2 X_{k}-X_{k-1}-X_{k+1}}\right)
$$

When using Hann's window, the authors use empirically determined correction coefficients P resp. Q for various window variations including Hann's window [20]. In this case, $\mathrm{Q}=0.55$ is recommended for the calculation of the $\delta$ complex value of the DFT spectrum by equation

$$
\delta=\operatorname{Re}\left(Q \frac{X_{k-1}-X_{k+1}}{2 X_{k}+X_{k-1}+X_{k+1}}\right)
$$

The second method uses only the modules of three components $\left|X_{k-1}\right|$ to $\left|X_{k+1}\right|$, where the original work was corrected for the shape in [20]

$$
\delta=P \frac{\left|X_{k+1}\right|-\left|X_{k-1}\right|}{\left|X_{k}\right|+\left|X_{k+1}\right|+\left|X_{k-1}\right|}
$$

where $P=1.36$ is recommended for Hann's window.

It turned out, however, that by simple correction of Eq. (10), when the signs of both smaller terms in the denominator change analogously to Eq. (9) and if we use a multiplication coefficient with the value exactly 2 , we get the relation 


$$
\delta=\operatorname{Re}\left(2 \frac{X_{k-1}-X_{k+1}}{2 X_{k}-X_{k-1}-X_{k+1}}\right)
$$

This adjusted relation achieves significantly higher accuracy of estimation $\delta$ (see Fig. $6 \mathrm{~b}$ than the most commonly used Jacobson's estimator for the rectangular window according to Eq. (9), see Fig. 6a.

Analogously to the correction of Eq. (10), the correction of Eq. (11) was also performed. Here the value of $\left|X_{\mathrm{k}}\right|$ was doubled and the exact coefficient 2 was again used for the whole value of $\delta$. The resulting relationship is

$$
\delta=2 \frac{\left|X_{k+1}\right|-\left|X_{k-1}\right|}{2\left|X_{k}\right|+\left|X_{k+1}\right|+\left|X_{k-1}\right|}
$$

Again, the resulting relationship gives a very low estimate error value, as shown in Fig. 6b. The results are practically the same as for Eq. (12) with the complex components. By comparing Eq. (13) with the available literature, it can be concluded that Agrež has worked towards the same relationship [19].
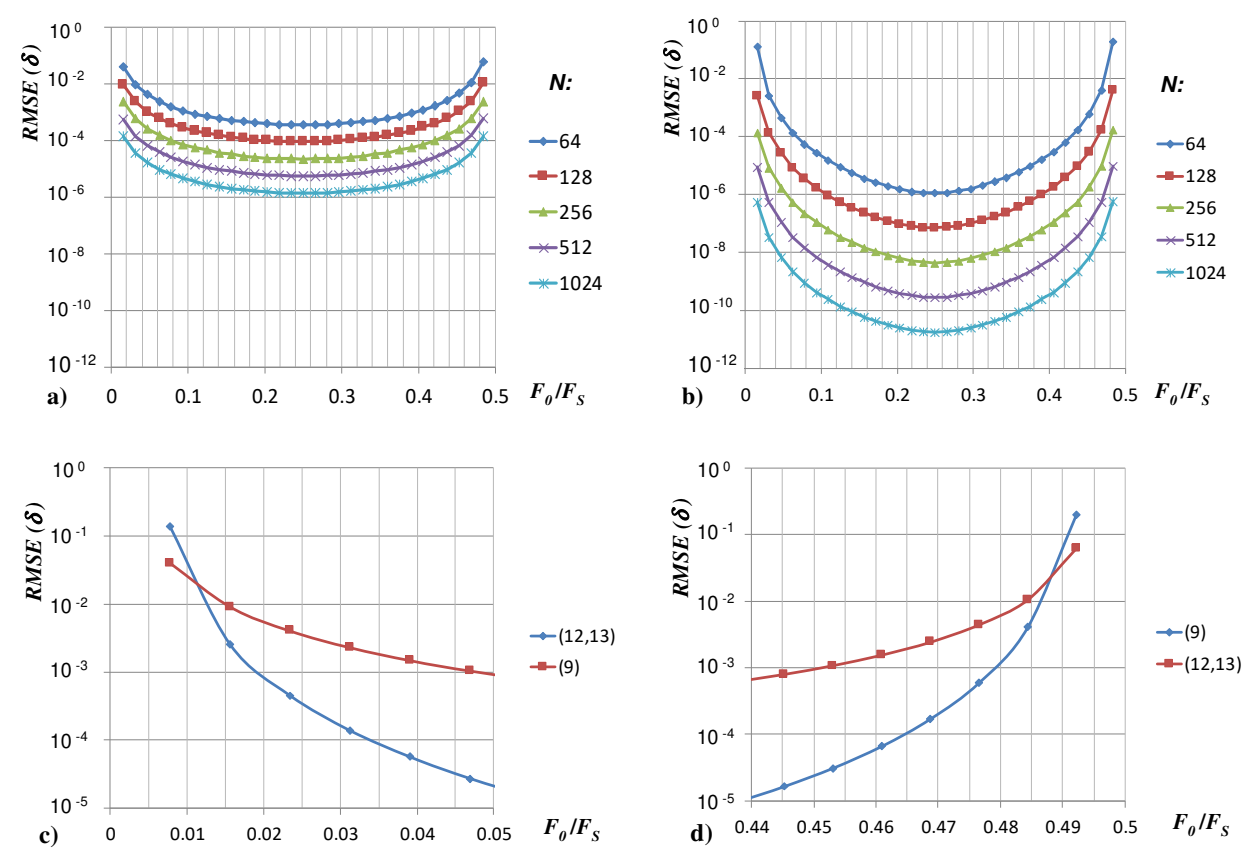

Fig. 6 RMSE of $\delta$ vs. $F_{0} / F_{S}$ for $\delta=0.25$ and for $N=64,128,256,512$ and 1024 a) relation (9), b) Eqs (12) and (13), comparison of Eq. (9) and Eqs (12) and (13) for $N=64$ c) for low value of $F_{0} / F_{S}(<0.05)$, d) for high value of $F_{0} / F_{S}(>0.44)$

Another possible way to reduce the instability of the deviations from the sinc function is to eliminate the difficult-to-solve effect of the undefined phase $\varphi_{0}$ of the test signal against the DFT window. It appears almost as random noise even for the amplitudes of the evaluated components, as explained in the analysis. Therefore, the solution is to use a time synchronization of the DFT window with the tested signal so that the phase $\varphi_{0}$ is defined and constant. 
The presented analysis shows that for a complex valued sinusoidal signal most of the above problems disappear. Neither changing the $F_{0} / F_{\mathrm{S}}$ value nor $\varphi_{0}$ value of the test signal is of any effect. The deviation of the DFT spectrum from the sinc function is only given by the value $N$. Therefore, it can be expressed explicitly and a zero estimate error can be obtained [15]. That is why it is preferable to use only a rectangular window.

The paper further shows that the solution of frequency estimation can be based on the estimation for the sinc spectrum itself without the effect of aliasing Eq. (9) according to Eq. (3).

\section{Conclusions}

The presented model of the DFT spectrum of the incoherent sinusoidal signal shows aliasing from all partial spectra from the $-F_{0}$ and $+F_{0}$ sub-bands as the cause of leakage deviations from sinc. In the case of a real valued sinusoidal signal, the essential influence of ratio $F_{0} / F_{\mathrm{S}}$ is explained. This aliasing and the phase shift of the signal $\varphi_{0}$ together with the phase jump by $\pi$ between the two parts of the basic spectrum (see Fig. 5) cause an unpleasant sensitivity of the spectrum module to the phase of the analyzed signal. This effect appears as a practical problem, for example when estimating the frequency of a dominant signal in the DFT analysis for many measurement tasks.

By contrast, in the case of a complex valued sinusoidal signal, it is only aliasing from the sub-bands for the frequencies $+F_{0}$, so that the above problems do not occur. The effect of aliasing is constant and defined only by value of $N$. This confirms the possibility of expressing an exact relationship for deviation $\delta(7,8)[15]$.

The ways to suppress these effects for a real valued sinusoidal signal result from this analysis and consist in suppressing the effect of aliasing including the effect of phase uncertainty. The easiest and well known way is to increase the value of $N$, but this is limited in practice. If possible, the discussed effect can further be reduced by setting the $F_{0} / F_{\mathrm{S}}$ ratio close to 0.25 in order to minimize the sum of aliasing from both sides.

Yet another way is to use a window function that suppresses the effective leakage band and thus the aliasing effect even for low $N$ values. This way was used in many different approaches with ambiguous or even contradictory results. The optimal choice of the window used depends on the $F_{0} / F_{\mathrm{S}}$ and SNR values, as can be deduced from the analysis. These parameters affect both the degree of the aliasing effect from individual sub-bands on deviations from the sinc function and the accuracy of their evaluation. Therefore, different estimation results for different values of these parameters could be achieved in the cited publications. On the other hand, some authors used unsuitable procedures where the estimated value $\delta$, calculated for a rectangular window, was used for other window types only with a fixed correction. These procedures appear to be inappropriate for the above reasons. Moreover, these relationships were corrected for the Hann's window. The resulting estimates for $\delta(12)$, (13) give high estimation accuracy $\delta$ in accordance with the expected effect of a suitably selected window.

Another possible way to minimize the analyzed effects is to synchronize the DFT window with the defined phase $\varphi_{0}$. In this way, the leakage deviations from the sinc function caused by a random change in the value of $\varphi_{0}$ can be suppressed as illustrated in Fig. 3 for a suitably selected $\varphi_{0}$ that results in a minimum estimate error value. 
In the case of complex sinusoidal signal aliasing, the effect of aliasing can be defined explicitly and there is no need to use special procedures for its minimization, as is the case with a real sine wave signal.

\section{Acknowledgement}

For the research, the infrastructure of the Department of Electrical Engineering, University of Defence in Brno, was used.

\section{References}

[1] OPPENHEIM, A.V. and SCHAFER, R.V. Discrete-Time Signal Processing. $3^{\text {rd }}$ ed. London: Pearson, 2009. 1144 p. ISBN 978-0-13-198842-2.

[2] LYONS, R.G. Understanding Digital Signal Processing. $2^{\text {nd }}$ ed. New Jersey: Prentice Hall, 2004. 688 p. ISBN 978-0-13-108989-1.

[3] SMITH, S.W. The Scientist and Engineer's Guide to Digital Signal Processing. $2^{\text {nd }}$ ed. San Diego: California Technical Publishing, 1999. 664 p. ISBN 978-0-96-601767-0.

[4] ROBERTS, M.J. Signals and Systems: Analysis Using Transform Methods and MATLAB®. New York: McGraw Hill, 2003. 1072 p. ISBN 978-0-07-249942-1.

[5] ZIELIŃSKI, T.P. and DUDA, K. Frequency and Damping Estimation Methods an Overview. Metrology and Measurement Systems, 2011, vol. 18, no. 4, p. 505-528. DOI 10.2478/v10178-011-005-y.

[6] DJUKANOVIĆ, S. An Accurate Method for Frequency Estimation of a Real Sinusoid. IEEE Signal Processing Letters, 2016, vol. 23, no. 7, p. 915-918. DOI 10.1109/LSP.2016.2564102.

[7] LUO, J., HOU, S., LI, X., OUYANG, Q. and ZHANG, Y. Generalization of Interpolation DFT Algorithms and Frequency Estimators with high Image Component Interference Rejection. EURASIP Journal on Advances in Signal Processing, 2016, Article number 30. DOI 10.1186/s13634-016-0330-6.

[8] BELEGA, D., PETRI, D. and DALLET, D. Frequency Estimation of a Sinusoidal Signal via a Three-Point Interpolated DFT Method with High Image Component Interference Rejection Capability. Digital Signal Processing, 2014, vol. 24, p. 162-169. DOI 10.1016/j.dsp.2013.09.014.

[9] RADIL, T., RAMOS, P.M. and SERRA, A.C. New Spectrum Leakage Correction Algorithm for Frequency Estimation of Power System Signals. IEEE Transactions on Instrumentation and Measurement, 2009, vol. 58, no. 5, p. 1670-1679. DOI 10.1109/TIM.2009.2014506.

[10] CANDAN, C. A Method for Fine Resolution Frequency Estimation from Three DFT Samples. IEEE Signal Processing Letters, 2011, vol. 18, no. 6, p. 351-354. DOI 10.1109/LSP.2011.2136378

[11] CANDAN, C. Analysis and Further Improvement of Fine Resolution Frequency Estimation Method from Three DFT Samples. IEEE Signal Processing Letters, 2013, vol. 20, no. 9, p. 913-916. DOI 10.1109/LSP.2013.2273616.

[12] CANDAN, C. Fine Resolution Frequency Estimation from Three DFT Samples: Case of Windowed Data. Signal Processing, 2015, vol. 114, p. 245-250. DOI 10.1016/j.sigpro.2015.03.009. 
[13] LIAO, J.-R. and LO, S. Analytical Solutions for Frequency Estimators by Interpolation of DFT Coefficients. Signal Processing, 2014, vol. 100, p. 93-100. DOI 10.1016/j.sigpro.2014.01.012.

[14] LIAO, J.-R. and CHEN, C.-M. Phase Correction of Discrete Fourier Transform Coefficients to Reduce Frequency Estimation Bias of Single Tone Complex Sinusoid. Signal Processing, 2014, vol. 94, p. 108-117. DOI 10.1016/j.sigpro.2013.05.021.

[15] XIANG, J., CUI, W. and SHEN, Q. Flexible and Accurate Frequency Estimation for Complex Sinusoid Signal by Interpolation Using DFT Samples. Chinese Journal of Electronics, 2018, vol. 27, no. 1, p. 109-114. DOI 10.1049/cje.2017.09.019.

[16] DUDA, K. and BARCZENTEWICZ, S. Interpolated DFT for $\sin \alpha(\mathrm{x})$ Windows. IEEE Transactions on Instrumentation and Measurement, 2014, vol. 63, no. 4, p. 754-760. DOI 10.1109/TIM.2013.2285795.

[17] DUDA, K. DFT Interpolation Algorithm for Kaiser-Bessel and Dolph-Chebyshev Windows. IEEE Transactions on Instrumentation and Measurement, 2011, vol. 60, no. 3, p. 784-790. DOI 10.1109/TIM.2010.2046594.

[18] WERNER, K.J. and GERMAIN, F.G. Sinusoidal Parameter Estimation Using Quadratic Interpolation around Power-Scaled Magnitude Spectrum Peaks. Applied Sciences, 2016, vol. 6, no. 10, p. 1-22. DOI 10.3390/app6100306.

[19] AGREŽ, D. Weighted Multipoint Interpolated DFT to Improve Amplitude Estimation of Multifrequency Signal. IEEE Transactions on Instrumentation and Measurement, 2002, vol. 51, no. 2, p. 287-292. DOI 10.1109/19.997826.

[20] JACOBSEN, E. and KOOTSOOKOS, P. Fast, Accurate Frequency Estimators. IEEE Signal Processing Magazine, 2007, vol. 24, no. 3, p. 123-125. DOI 10.1109/ MSP.2007.361611.

[21] CHEN, H., XU, F. an LI, J. A Frequency Estimator for Real Valued Sinusoidal Signals Using Three DFT Samples. In Proceedings of the 2018 International Conference on Radar (RADAR). Brisbane: IEEE, 2018. DOI 10.1109/RADAR.2018.8557341. 